\title{
The Multiple Influences of Nickel \\ Concentration Gradient Structure and \\ Yttrium Element Substitution on the \\ Structural and Electrochemical \\ Performances of $\mathrm{NaNi}_{0.25} \mathrm{Mn}_{0.25} \mathrm{Fe}_{0.5} \mathrm{O}_{2}$ \\ Cathode Material
}

Kang Wu, ${ }^{a, b, c} \mathrm{Na} \mathrm{Li},{ }^{d}$ Kuanrong Hao, ${ }^{d}$ Wen Yin ${ }^{f}$ Min Wang, ${ }^{a, b}$ Guofeng Jia ${ }^{a, b}, \mathrm{Yu}$ Lin Lee, ${ }^{g}$ Rongbin Dang, ${ }^{d}$ Xin Deng, ${ }^{d}$ Xiaoling Xiao ${ }^{* d}$, Enyue Zhao ${ }^{* e}$ and Zhijian

$$
W u^{* a, b}
$$

${ }^{a}$ Key Laboratory of Comprehensive and Highly Efficient Utilization of Salt Lake Resources, Qinghai Institute of Salt Lakes, Chinese Academy of Sciences, 18th Xinning Road, Xining 810008, China.

${ }^{b}$ Key Laboratory of Salt Lake Resources Chemistry of Qinghai Province, Xining 810008, China.

${ }^{c}$ University of Chinese Academy of Sciences, Beijing 100049, China.

${ }^{d}$ College of Materials Science and Opto-electronic Technology, Center of Materials Science and Optoelectronics Engineering, University of Chinese Academy of Sciences, Beijing 100049, China.

${ }^{e}$ Songshan Lake Materials Laboratory, Dongguan, 523808, China.

${ }^{f}$ Spallation Neutron Source Science Center, Dongguan, 523803, PR China.

${ }^{g}$ Department of Materials, Imperial College London, Royal School of Mines, Exhibition Road, London SW7 2AZ, UK. 




Figure S1. XRD patterns of NMF, NCG-NMF and NCG-NMF-Y materials.
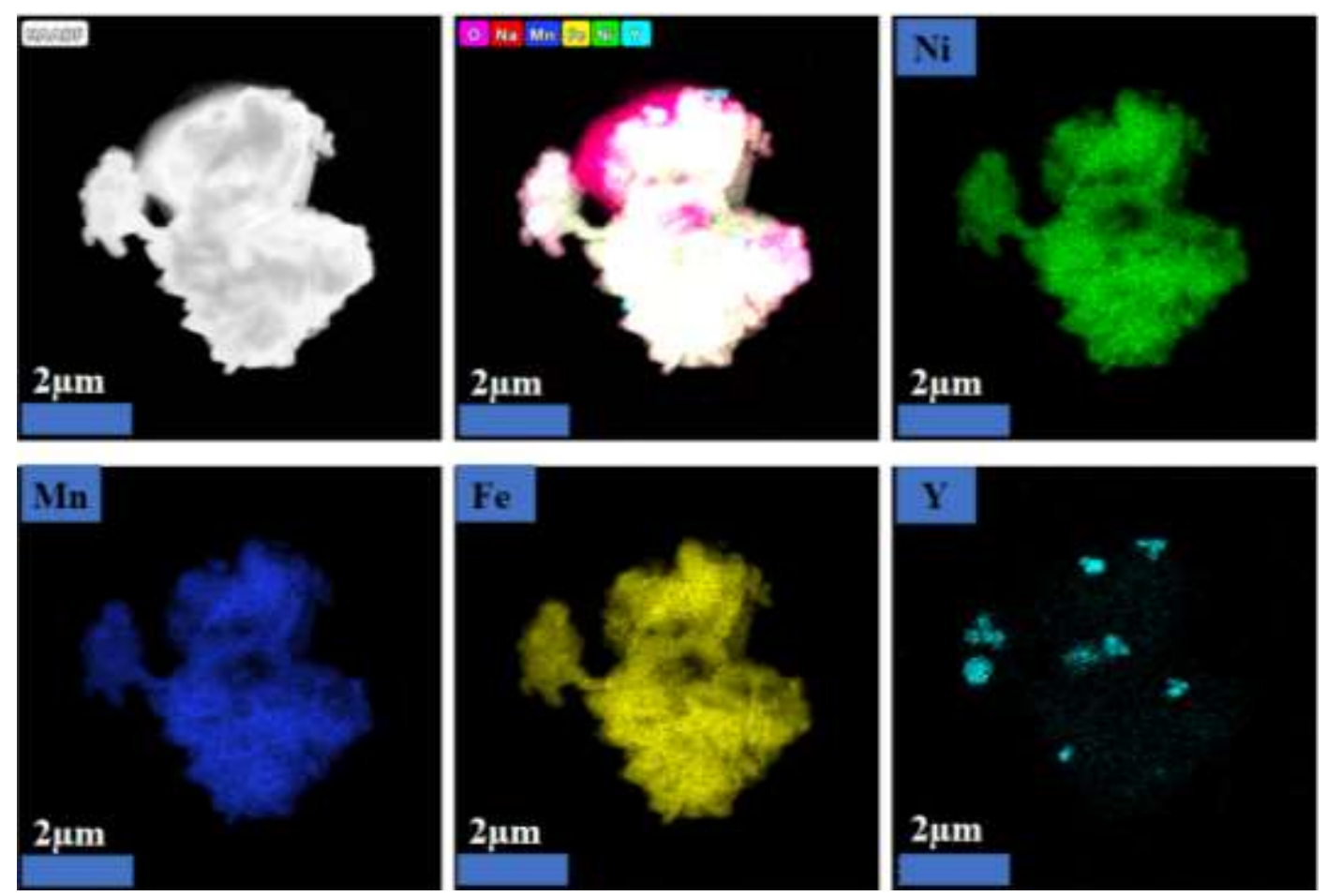

Figure S2. The high-resolution transmission electron microscope images of NCG-NMF-Y and the corresponding EDX elemental mappings (Ni, Mn, Fe, Y). 

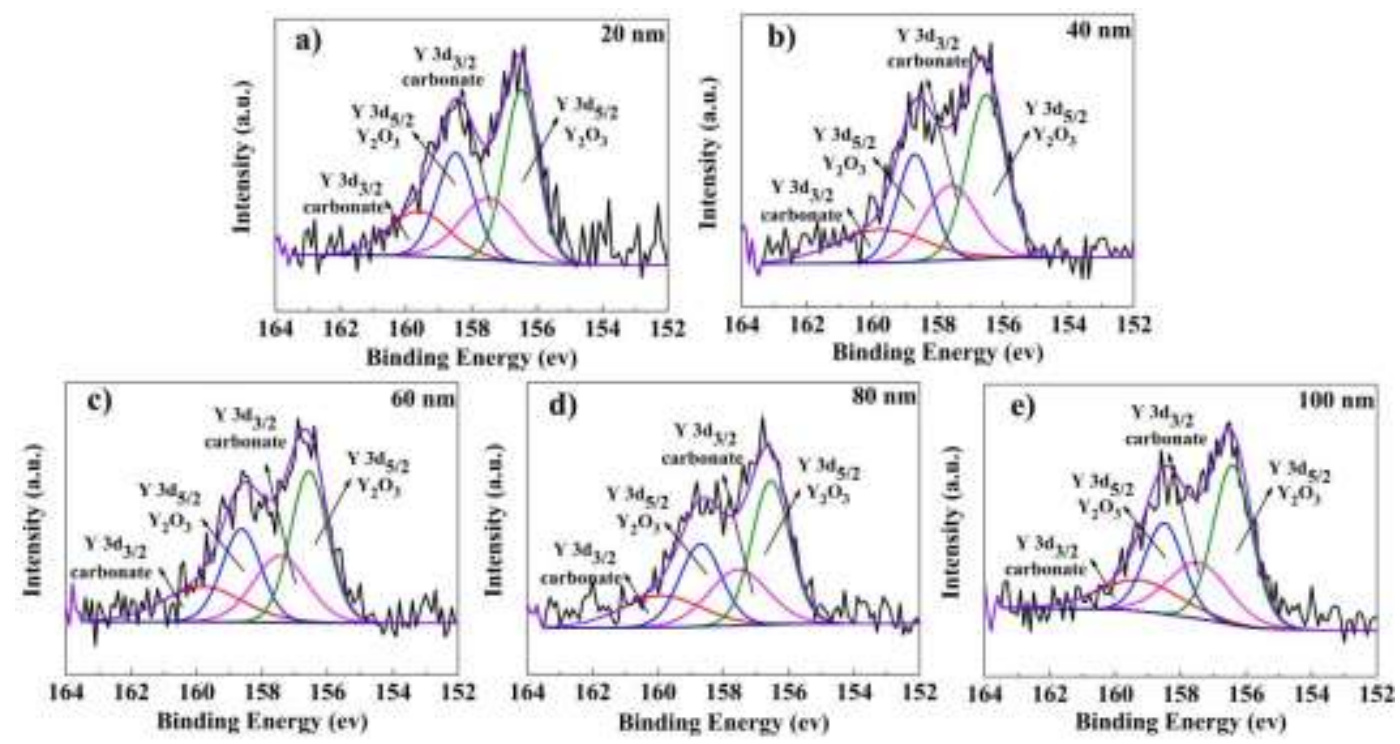

Figure S3. X-ray photoelectron spectroscopy (XPS) spectra of Y element for NCG-NMF-Y material etched at $20 \mathrm{~nm}, 40 \mathrm{~nm}, 60 \mathrm{~nm}, 80 \mathrm{~nm}$ and $100 \mathrm{~nm}$.
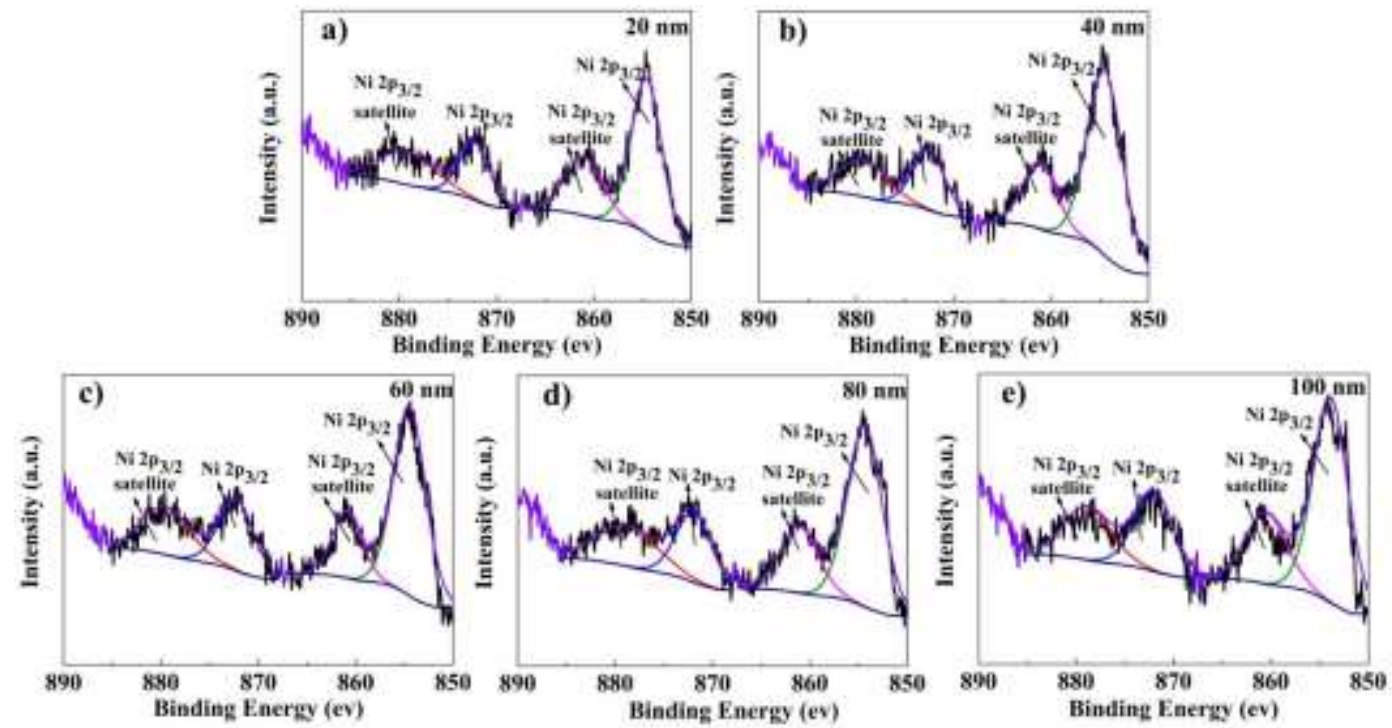

Figure S4. X-ray photoelectron spectroscopy (XPS) spectra of Ni element for NCG-NMF-Y material etched at $20 \mathrm{~nm}, 40 \mathrm{~nm}, 60 \mathrm{~nm}, 80 \mathrm{~nm}$ and $100 \mathrm{~nm}$. 

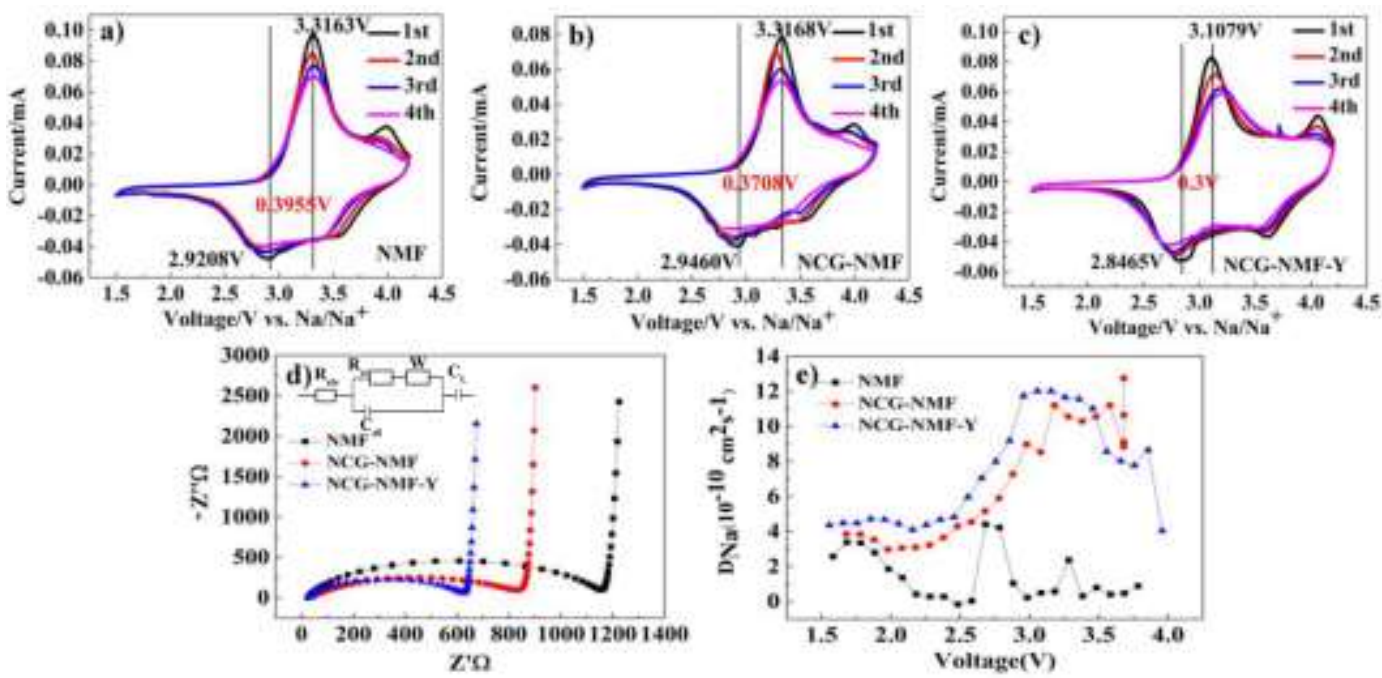

Figure S5. CV curves of the NMF a), NCG-NMF b) and NCG-NMF-Y c) electrodes at a scan rate of $0.1 \mathrm{mV} \mathrm{s}^{-1}$. d) Nyquist plots for the NMF, NCG-NMF and NCG-NMF-Y electrodes before cycling. The inset image shows related equivalent circuit. e) $\mathrm{Na}$ diffusion coefficients $\left(\mathrm{DNa}_{\mathrm{Na}}^{+}\right)$calculated from the PITT curves versus voltage during discharge processes for the NMF, NCG-NMF and NCG-NMF-Y electrodes.
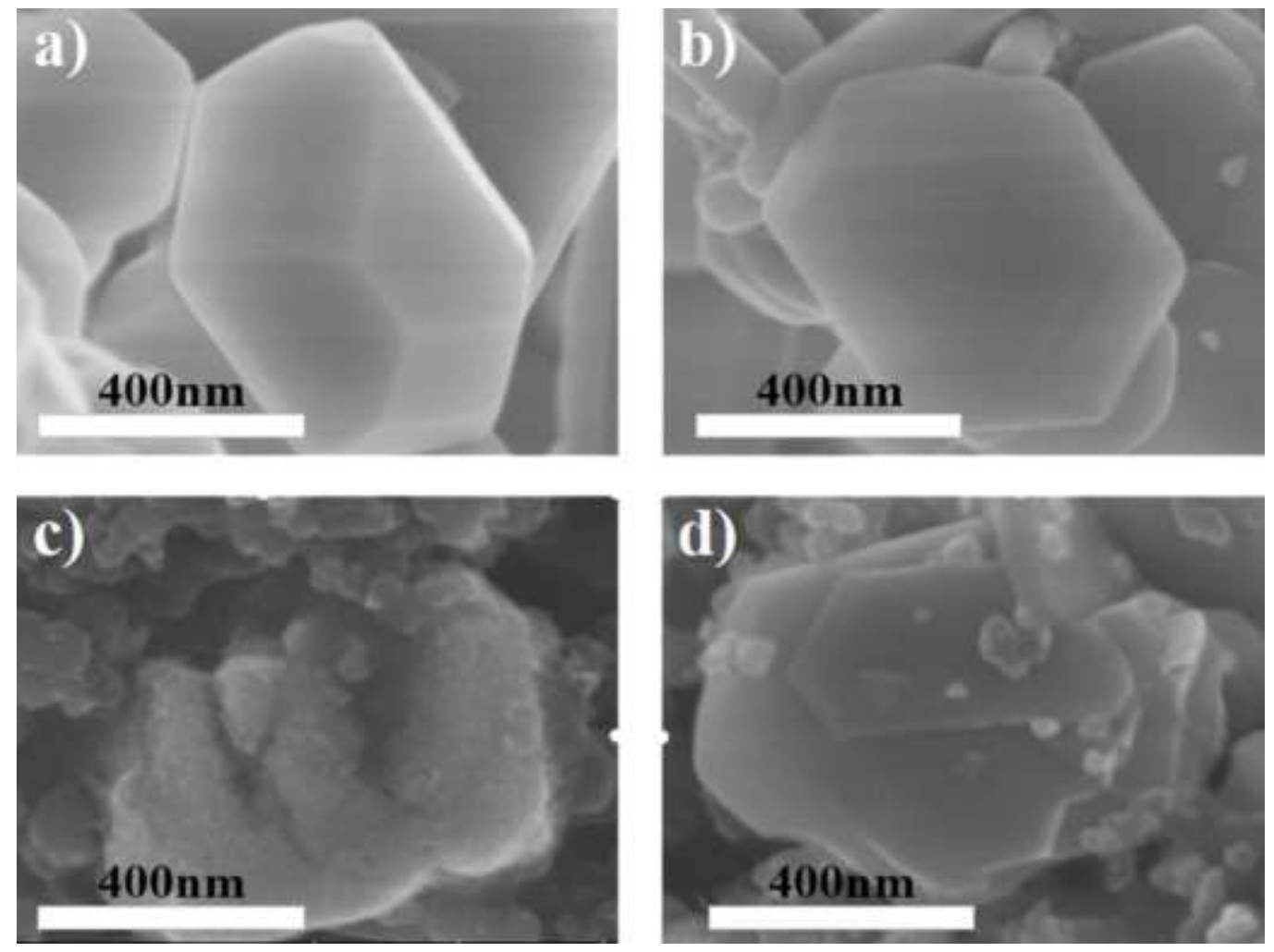

Figure S6. SEM images of a,c) pristine and b,d) NCG-NMF-Y samples collected at different stages: a,b) as-synthesized, c,d) at $1 \mathrm{C}$-rate for 100 cycles. 


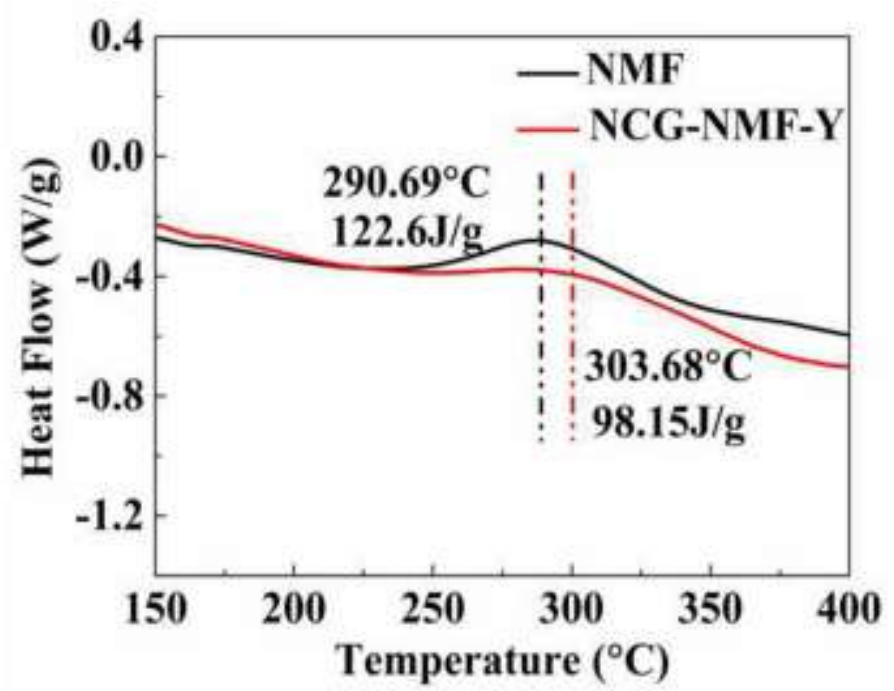

Figure S7. DSC profiles of NMF and NCG-NMF-Y electrodes at charged state of 4.2 V. 
Table S1. The bond energies $\left(\mathrm{kJ} \mathrm{mol}^{-1}\right)$ between metallic elements and oxygen.

\begin{tabular}{ccccccccccc}
\hline M-O & Ni-O & Mn-O & Fe-O & Sb-O & Y-O & Cr-O & Cu-O & Bi-O & Sc-O & Al-O \\
\hline $\begin{array}{c}\text { Bond } \\
\begin{array}{c}\text { Energy } \\
\mathrm{kJ} \mathrm{mol}^{-1}\end{array}\end{array}$ & 391 & 465 & 409 & 372 & 715 & 427 & 343 & 343 & 674 & 512 \\
\hline
\end{tabular}

Table S2. The refined crystal structure of the pristine NMF using neutron diffraction data.

S.G. R-3m a $=\mathrm{b}=2.9914(1) \AA, c=16.0290(5) \AA, \alpha=\beta=90^{\circ}, \gamma=120^{\circ} \mathrm{NaO}_{2}=3.226$ $\AA, \mathrm{TMO}_{2}=2.117 \AA$

\begin{tabular}{lllllll}
\hline Atom & Wyck. & $\mathrm{x}$ & $\mathrm{y}$ & $\mathrm{z}$ & Occ. & $U_{\text {iso }}\left(\AA^{2}\right)$ \\
\hline $\mathrm{Na}$ & $3 \mathrm{a}$ & 0 & 0 & 0 & 1 & $0.0144(3)$ \\
$\mathrm{Mn}$ & $3 \mathrm{~b}$ & 0 & 0 & 0.5 & 0.25 & $0.0039(3)$ \\
$\mathrm{Ni}$ & $3 \mathrm{~b}$ & 0 & 0 & 0.5 & 0.25 & $0.0039(3)$ \\
$\mathrm{Fe}$ & $3 \mathrm{~b}$ & 0 & 0 & 0.5 & 0.5 & $0.0039(3)$ \\
$\mathrm{O}$ & $6 \mathrm{c}$ & 0 & 0 & $0.23269(3)$ & 1 & $0.0116(2)$ \\
\hline
\end{tabular}

Table S3. The refined crystal structure of the NCG-NMF using neutron diffraction data.

\begin{tabular}{|c|c|c|c|c|c|c|}
\hline \multicolumn{7}{|c|}{$\begin{array}{l}\text { S.G. R-3m a }=b=2.9891(2) \AA, c=16.0388(5) \AA, \alpha=\beta=90^{\circ}, \gamma=120^{\circ} \mathrm{NaO}_{2}=3.231 \\
\AA, \mathrm{TMO}_{2}=2.115 \AA\end{array}$} \\
\hline \multicolumn{7}{|c|}{$\begin{array}{lllll}\text { Site } & \text { Wyck. } & \text { x } & \text { y } & \text { z }\end{array}$} \\
\hline \multicolumn{7}{|c|}{$\begin{array}{lllllll}\text { Site } & \text { Wyck. } & \mathrm{x} & \mathrm{y} & \mathrm{z} & \text { Occ. } & U_{\text {iso }}\left(\AA^{2}\right) \\
\mathrm{Na} & 3 \mathrm{a} & 0 & 0 & 0 & 1 & 0.0152(4)\end{array}$} \\
\hline \multicolumn{7}{|l|}{$\mathrm{Mn}$} \\
\hline \multirow{2}{*}{$\begin{array}{l}\mathrm{Ni} \\
\mathrm{Fe}\end{array}$} & $3 b$ & 0 & 0 & 0.5 & 0.25 & $0.0051(3)$ \\
\hline & $3 b$ & 0 & 0 & 0.5 & 0.5 & $0.0051(3)$ \\
\hline $\mathrm{O}$ & $6 c$ & 0 & 0 & $0.23258(4)$ & 1 & $0.0116(3)$ \\
\hline
\end{tabular}

Table S4. The refined crystal structure of the NCG-NMF-Y using neutron diffraction data.

S.G. R-3m a $=b=2.9870(2) \AA, c=16.0353(6) \AA, \alpha=\beta=90^{\circ}, \gamma=120^{\circ} \mathrm{NaO}_{2}=3.234$ $\AA, \mathrm{TMO}_{2}=2.111 \AA$

\begin{tabular}{lllllll}
\hline Atom & Wyck. & $\mathrm{x}$ & $\mathrm{y}$ & $\mathrm{z}$ & Occ. & $U_{\text {iso }}\left(\AA^{2}\right)$ \\
\hline $\mathrm{Na}$ & $3 \mathrm{a}$ & 0 & 0 & 0 & 1 & $0.0145(5)$ \\
$\mathrm{Mn}$ & $3 \mathrm{~b}$ & 0 & 0 & 0.5 & $0.236(4)$ & $0.0054(4)$ \\
$\mathrm{Ni}$ & $3 \mathrm{~b}$ & 0 & 0 & 0.5 & $0.262(3)$ & $0.0054(4)$ \\
$\mathrm{Fe}$ & $3 \mathrm{~b}$ & 0 & 0 & 0.5 & $0.485(2)$ & $0.0054(4)$ \\
$\mathrm{O}$ & $6 \mathrm{c}$ & 0 & 0 & $0.23250(4)$ & 1 & $0.0099(3)$ \\
$\mathrm{Y}$ & $3 \mathrm{~b}$ & 0 & 0 & 0.5 & $0.017(1)$ & $0.0054(4)$ \\
\hline
\end{tabular}


Table S5. The ICP results of NMF, NCG-NMF and NCG-NMF-Y materials.

\begin{tabular}{cccc}
\hline Sample & $\mathrm{Ni}$ & $\mathrm{Mn}$ & $\mathrm{Fe}$ \\
\hline NMF & 0.28 & 0.23 & 0.5 \\
NCG-NMF & 0.28 & 0.25 & 0.5 \\
NCG-NMF-Y & 0.28 & 0.25 & 0.5 \\
\hline
\end{tabular}

Table S6. The peak area of Ni and Y elements for NCG-NMF-Y material etched at 20 $\mathrm{nm}, 40 \mathrm{~nm}, 60 \mathrm{~nm}, 80 \mathrm{~nm}$ and $100 \mathrm{~nm}$.

\begin{tabular}{|c|c|c|c|c|c|}
\hline Peak/Area & $20 \mathrm{~nm}$ & $40 \mathrm{~nm}$ & $60 \mathrm{~nm}$ & $80 \mathrm{~nm}$ & $100 \mathrm{~nm}$ \\
\hline $\mathrm{Y} 3 \mathrm{~d}_{3 / 2}$ (carbonate) & 312 & 362 & 369 & 373 & 370 \\
\hline $\mathrm{Y} 3 \mathrm{~d}_{5 / 2}\left(\mathrm{Y}_{2} \mathrm{O}_{3}\right)$ & 437 & 459 & 486 & 509 & 516 \\
\hline $\mathrm{Y} 3 \mathrm{~d}_{3 / 2}$ (carbonate) & 406 & 472 & 476 & 486 & 506 \\
\hline $\mathrm{Y} 3 \mathrm{~d}_{5 / 2}\left(\mathrm{Y}_{2} \mathrm{O}_{3}\right)$ & 644 & 766 & 744 & 751 & 900 \\
\hline $\mathrm{Ni} 2 \mathrm{p}_{3 / 2}$ (satellite) & 2670 & 2592 & 3380 & 3907 & 3739 \\
\hline $\mathrm{Ni} 2 \mathrm{p}_{3 / 2}$ & 2580 & 2808 & 3677 & 3923 & 4517 \\
\hline $\mathrm{Ni} 2 \mathrm{p}_{3 / 2}$ (satellite) & 3187 & 3318 & 2910 & 4003 & 4303 \\
\hline $\mathrm{Ni} 2 \mathrm{p}_{3 / 2}$ & 6940 & 9464 & 8877 & 10023 & 10470 \\
\hline
\end{tabular}

Table S7. The Y and Ni proportion for NCG-NMF-Y material etched at $20 \mathrm{~nm}, 40$ $\mathrm{nm}, 60 \mathrm{~nm}, 80 \mathrm{~nm}$ and $100 \mathrm{~nm}$.

\begin{tabular}{cccccc}
\hline Depth & $20 \mathrm{~nm}$ & $40 \mathrm{~nm}$ & $60 \mathrm{~nm}$ & $80 \mathrm{~nm}$ & $100 \mathrm{~nm}$ \\
\hline Y atomic\% & 0.41 & 0.46 & 0.46 & 0.47 & 0.5 \\
Ni atomic\% & 1.98 & 2.13 & 2.2 & 2.43 & 2.6 \\
\hline
\end{tabular}

Table S8. The binding energies (eV) of Ni2 $\mathrm{p}_{3 / 2}, \mathrm{Mn} 2 \mathrm{p}_{3 / 2}, \mathrm{Fe} 2 \mathrm{p}_{3 / 2}$ and peak areas of $\mathrm{Mn}^{4+} 2 \mathrm{p}_{3 / 2}, \mathrm{Fe}^{3+} 2 \mathrm{p}_{3 / 2}$ for the NMF, NCG-NMF and NCG-NMF-Y materials.

\begin{tabular}{cccc}
\hline Sample & NMF & NCG-NMF & NCG-NMF-Y \\
\hline $\mathrm{Ni}^{2+} 2 \mathrm{p}_{3 / 2}(\mathrm{eV})$ & 855.1 & 855.1 & 855.16 \\
$\mathrm{Mn}^{4+} 2 \mathrm{p}_{3 / 2}(\mathrm{eV})$ & 642.549 & 642.545 & 642.253 \\
$\mathrm{Fe}^{3+} 2 \mathrm{p}_{3 / 2}(\mathrm{eV})$ & 710.956 & 711.096 & 711.106 \\
\hline
\end{tabular}

Table S9. The simulation results of EIS of NMF and NCG-NMF-Y materials.

\begin{tabular}{cccc}
\hline Samples & NMF & NCG-NMF & NCG-NMF-Y \\
\hline$R_{\text {ele }}(\Omega)$ & 24.8 & 23.1 & 22.2 \\
$R_{\mathrm{ct}}(\Omega)$ & 1138.1 & 850.4 & 609.9 \\
Estimated error/R $\mathrm{R}_{\mathrm{ele}}(\%)$ & 2.1 & 2.2 & 2.3 \\
Estimated error/Rct $(\%)$ & 1.4 & 1.3 & 1.6 \\
\hline
\end{tabular}


Table S10. The amount of dissolved Ni, Mn and Fe ions of NMF and NCG-NMF-Y cathode materials.

\begin{tabular}{ccccc}
\hline Storage time & Samples & $\mathrm{Ni}\left(\mathrm{mg} \mathrm{kg}^{-1}\right)$ & $\mathrm{Mn}\left(\mathrm{mg} \mathrm{kg}^{-1}\right)$ & $\mathrm{Fe}\left(\mathrm{mg} \mathrm{kg}^{-1}\right)$ \\
\hline 15days & NMF & 0.003661 & 0.001569 & 0.0086295 \\
5ml & NCG-NMF-Y & 0.0028765 & 0.001569 & 0.0044455 \\
\hline
\end{tabular}

